\title{
THE DISTRIBUTION OF NONPRINCIPAL EIGENVALUES OF SINGULAR SECOND-ORDER LINEAR ORDINARY DIFFERENTIAL EQUATIONS
}

JUAN PABLO PINASCO

Received 21 February 2005; Accepted 27 July 2006

We obtain the asymptotic distribution of the nonprincipal eigenvalues associated with the singular problem $x^{\prime \prime}+\lambda q(t) x=0$ on an infinite interval $[a,+\infty)$. Similar to the regular eigenvalue problem on compact intervals, we can prove a Weyl-type expansion of the eigenvalue counting function, and we derive the asymptotic behavior of the eigenvalues.

Copyright (c) 2006 Hindawi Publishing Corporation. All rights reserved.

\section{Introduction}

In this work we study the second-order linear ordinary differential equation

$$
x^{\prime \prime}+\lambda q(t) x=0, \quad t \geq a,
$$

with the boundary conditions

$$
x(a, \lambda)=0, \quad \lim _{t \rightarrow \infty}[x(t, \lambda)-t]=0, \quad \lim _{t \rightarrow \infty} t\left[x^{\prime}(t, \lambda)-1\right]=0,
$$

where $\lambda$ is a real parameter and $q(t)$ is a positive continuous function on $[a, \infty)$ satisfying

$$
\int_{a}^{\infty} t^{2} q(t) d t<\infty
$$

A nonoscillatory solution $x_{0}(t, \lambda)$ of (1.1) satisfying the boundary conditions (1.2) is called a nonprincipal eigenfunction if

$$
\int_{a}^{\infty} \frac{d t}{\left(x_{1}(t, \lambda)\right)^{2}}<\infty
$$

and the corresponding value of $\lambda$ is called a nonprincipal eigenvalue. 
Concerning the existence and uniqueness of nonprincipal eigenvalues, the main result is due to Elbert et al. [2]. There exists a sequence of positive constants $\left\{\lambda_{k}\right\}_{k}, 0 \leq \lambda_{0}<\lambda_{1}<$ $\cdots<\lambda_{k}<\cdots>\infty$ such that, for each $\lambda=\lambda_{k}$, (1.1) possesses a solution $x_{k}\left(t, \lambda_{k}\right)$ satisfying the boundary condition (1.2) and having exactly $k$ zeros in $(a, \infty), k=0,1,2, \ldots$, imposing the integrability condition (1.3) on $q(t)$.

We are interested in the distribution and asymptotic behavior of eigenvalues $\left\{\lambda_{k}\right\}_{k}$. To this end, we study the spectral counting function

$$
N(\lambda)=\#\left\{k: \lambda_{k} \leq \lambda\right\} .
$$

It is well known that the eigenvalue problem in a closed interval $[a, b]$ has the asymptotic distribution (see [1]):

$$
N(\lambda) \sim \frac{\lambda^{1 / 2}}{\pi} \int_{a}^{b} q^{1 / 2}(t) d t
$$

as $\lambda \rightarrow \infty$, generalizing the Weyl formula. Here, $f \sim g$ means that $f / g \rightarrow 1$.

Our main result is the following theorem.

Theorem 1.1. Let $\left\{\lambda_{k}\right\}$ be the sequence of nonprincipal eigenvalues of problem (1.1)-(1.2), and let $q(t)$ be a positive, continuous, and nonincreasing function satisfying (1.3). Then, the asymptotic expansion of $N(\lambda)$ is given by

$$
N(\lambda)=\frac{\lambda^{1 / 2}}{\pi} \int_{a}^{\infty} q^{1 / 2}(t) d t+o\left(\lambda^{1 / 2}\right)
$$

as $\lambda \rightarrow \infty$. Also, the kth-eigenvalue has the following asymptotic behavior:

$$
\lambda_{k-1}=\left(\frac{\pi k}{\int_{a}^{\infty} q^{1 / 2}(t) d t}\right)^{2}+o\left(k^{2}\right)
$$

as $k \rightarrow \infty$.

The paper is organized as follows. In Section 2 we prove some auxiliary results, and the proof of Theorem 1.1 is given in Section 3.

\section{Sturm-Liouville bracketing of eigenvalues}

Let us observe that problem (1.1)-(1.2) is not a variational one, since $x^{\prime}(t) \sim 1$ as $t \rightarrow$ $+\infty$ and $x^{\prime}(t) \notin L^{2}(0,+\infty)$. Hence, we need the following generalization of the DirichletNeumann bracketing of Courant (see [1]) in order to prove Theorem 1.1.

THeOREM 2.1. Let $N(\lambda, I)$ be the spectral counting function on $I=(a, b)$ of the problem

$$
-x^{\prime \prime}=\lambda q(t) x, \quad x(a)=0=x(b) .
$$

Let $c \in(a, b)$. Then,

$$
N(\lambda, I) \sim N\left(\lambda, I_{1}\right)+N\left(\lambda, I_{2}\right)
$$

as $\lambda \rightarrow \infty$, where $I_{1}=(a, c)$ and $I_{2}=(c, b)$. 
Remark 2.2. For simplicity, we deal only with the Dirichlet boundary condition on a bounded interval. With minor modifications of the proof, the result is valid for different boundary conditions, including the case $b=+\infty$ and the boundary condition (1.2), since the proof is based on the Sturm-Liouville oscillation theory.

Let us sketch the proof of the Dirichlet Neumann bracketing for a second-order differential operator $L$ with variational structure in an interval $I$. The eigenvalues of $L$ are obtained minimizing a quadratic functional in a convenient subspace $H \subset H^{1}(I)$. We have

$$
H_{0}^{1}\left(I_{1}\right) \oplus H_{0}^{1}\left(I_{2}\right) \subset H_{0}^{1}(I) \subset H \subset H^{1}(I) \subset H^{1}\left(I_{1}\right) \oplus H^{1}\left(I_{2}\right)
$$

and we obtain the Dirichlet eigenvalues of $L$ in $I_{1}$ and $I_{2}$ as an upper bound of the eigenvalues of $L$ in $I$, and the Neumann eigenvalues of $I_{1}$ and $I_{2}$ as a lower bound.

In problem (1.1)-(1.2), the solutions and eigenvalues are obtained by a fixed point argument, instead of a minimization procedure, and we need a different argument to relate the eigenvalue of two intervals and those of the union of them. Since the eigenfunction $x_{k}$ has exactly $k$ zeros in $(a, b)$, it is possible to obtain the asymptotic distribution of eigenvalues from the asymptotic number of zeros of solutions, an idea which goes back at least to Hartman (see [3]). For the sake of self-completeness, we prove Theorem 2.1 here.

Proof of Theorem 2.1. Let us consider the following eigenvalue problems in $I_{1}$ and $I_{2}$, with the original boundary conditions in $a$ and $b$, and a Neumann boundary condition at $c$ :

$$
\begin{gathered}
-u^{\prime \prime}=\mu q(t) u, \quad t \in(a, c), \\
u(a)=0, \quad u^{\prime}(c)=0, \\
-v^{\prime \prime}=v q(t) v, \quad t \in(c, b), \\
v^{\prime}(c)=0, \quad v(b)=0 .
\end{gathered}
$$

For each problem there exists a sequence of simple eigenvalues $\left\{\mu_{k}\right\}_{k},\left\{\nu_{k}\right\}_{k}$ tending to infinity, and the $k$ th eigenfunction $u_{k}$ corresponding to $\mu_{k}$ (resp., $v_{k}, v_{k}$ ) has exactly $k$ zeros.

Let $\lambda$ be fixed. Let $\lambda_{n}$ be the greater eigenvalue of problem (2.1) lower or equal than $\lambda$ and $x_{n}(t)$ the corresponding eigenfunction, which has $n$ zeros in $(a, b)$. Let $k$ be the number of zeros of $x_{n}$ in $(a, c)$, and let $n-k$ be the number of zeros in $(c, b)$.

Let $\mu_{j}$ be the greater eigenvalue of problem (2.4) lower or equal than $\lambda$, and let $u_{j}$ be the corresponding eigenfunction. We will show that $j$, the number of zeros of $u_{j}$, satisfies

$$
k-1 \leq j \leq k+2
$$

Let us suppose first that $u_{j}$ has $k+3$ zeros. Then, the Sturmian theory gives $\mu_{j}>\lambda_{n}$. Let $x_{\mu_{j}}(t)$ be the unique solution of (2.1) satisfying

$$
\begin{aligned}
& x_{\mu_{j}}(c)=u_{j}(c), \\
& x_{\mu_{j}}^{\prime}(c)=u_{j}^{\prime}(c) .
\end{aligned}
$$


Hence, $x_{\mu_{j}} \equiv u_{j}$ in $(a, c)$, and $x_{\mu_{j}}(t)$ has at least $n-k-1$ zeros in $(c, b)$ (let us note that one of the original zeros of $x_{n}(t)$ could cross the point $c$ to the left). Thus, the solution $x_{\mu_{j}}(t)$ has at least $n+2$ zeros in $(a, b)$.

However, the eigenfunction $x_{n+1}(t)$ of problem (2.1) corresponding to the eigenvalue $\lambda_{n+1}$ has $n+1$ zeros and satisfy $\lambda_{n+1}<\mu_{j}$. Hence,

$$
\lambda_{n+1}<\mu_{j} \leq \lambda,
$$

which contradicts our assumption.

On the other hand, let us suppose that $u_{j}$ has $k-2$ zeros. Clearly, $\mu_{j}<\lambda_{n}<\lambda$. Let $u_{j+1}$ be the eigenfunction of problem (2.4) with $k-1$ zeros in $(a, c)$, and let $\mu_{j+1}$ be the corresponding eigenvalue. By using the Sturm-Liouville theory,

$$
\mu_{j+1}<\lambda_{n}<\lambda,
$$

because $x_{n}(t)$ has $k$ zeros in $(a, c)$, which contradicts the fact that $\mu_{j}$ is the greater eigenvalue of problem (2.4) lower or equal than $\lambda$.

Let us consider now problem (2.5). Let $\nu_{h}$ be the greater eigenvalue of problem (2.5) lower or equal than $\lambda$, and let $v_{h}$ be the corresponding eigenfunction. In much the same way, fixing the boundary condition at $t=b$, we can show that $h$, the number of zeros of $v_{h}$, satisfy

$$
n-k-2 \leq h \leq n-k+1
$$

Then, from inequalities (2.6) and (2.10),

$$
N\left(\lambda, I_{1}\right)+N\left(\lambda, I_{2}\right)-3 \leq N(\lambda, I) \leq N\left(\lambda, I_{1}\right)+N\left(\lambda, I_{2}\right)+3
$$

and the proof is finished.

\section{Asymptotic of nonprincipal eigenvalues}

In this section we prove Theorem 1.1. First, we need the following lemma.

LEMMA 3.1. Let $q(t)$ be a positive continuous function satisfying

$$
\int_{a}^{\infty} t^{2} q(t) d t<\infty
$$

Then,

$$
\int_{a}^{\infty} q^{1 / 2}(t) d t<\infty
$$

Proof. It follows from Holder's inequality:

$$
\int_{a}^{\infty} q^{1 / 2}(t) d t<\left(\int_{a}^{\infty} t^{2} q(t) d t\right)^{1 / 2}\left(\int_{a}^{\infty} t^{-2} d t\right)^{1 / 2}<\infty .
$$


We divide the proof of Theorem 1.1 in three parts. We obtain an optimal lower bound for $N(\lambda)$; then we obtain an upper bound for $N(\lambda)$; and finally, we improve the upper bound.

Proposition 3.2. Let $N(\lambda)$ be the eigenvalue counting function of Theorem 1.1. The following inequality holds:

$$
\frac{\lambda^{1 / 2}}{\pi} \int_{a}^{+\infty} q^{1 / 2}(t) d t+o\left(\lambda^{1 / 2}\right) \leq N(\lambda)
$$

Proof. Let $\varepsilon>0$ be fixed, there exist $T_{\varepsilon}$ such that

$$
\frac{1}{\pi} \int_{T_{\varepsilon}}^{\infty} q^{1 / 2}(t) d t \leq \frac{\varepsilon}{2}
$$

Let us consider the Dirichlet eigenvalue problem on $\left[a, T_{\varepsilon}\right]$ :

$$
\begin{gathered}
-y^{\prime \prime}(t)=\mu q(t) y(t), \\
y(a)=0=y\left(T_{\varepsilon}\right) .
\end{gathered}
$$

It is well known that there exists a sequence of eigenvalues $\left\{\mu_{k}\right\}_{k \geq 0}$, with associated eigenfunctions $\left\{y_{k}\right\}_{k \geq 0}$. Each eigenvalue is isolated and $y_{k}$ has exactly $k$ zeros in the open interval $\left(a, T_{\varepsilon}\right)$.

The spectral counting function $N_{D}\left(\lambda,\left[a, T_{\varepsilon}\right]\right)$ of problem (3.6) has the following asymptotic expansion:

$$
N_{D}\left(\lambda,\left[a, T_{\varepsilon}\right]\right)=\frac{\lambda^{1 / 2}}{\pi} \int_{a}^{T_{\varepsilon}} q^{1 / 2}(t) d t+o\left(\lambda^{1 / 2}\right)
$$

Therefore, for the same $\varepsilon>0$, there exists $\lambda(\varepsilon)$ such that

$$
\left|\frac{N_{D}\left(\lambda,\left[a, T_{\varepsilon}\right]\right)}{\lambda^{1 / 2}}-\frac{1}{\pi} \int_{a}^{T_{\varepsilon}} q^{1 / 2}(t) d t\right| \leq \frac{\varepsilon}{2}
$$

for every $\lambda \geq \lambda(\varepsilon)$.

By the Sturmian comparison theorem, we have the inequality $\lambda_{k} \leq \mu_{k}$, which gives the lower bound for $N(\lambda)$ :

$$
N_{D}\left(\lambda,\left[a, T_{\varepsilon}\right]\right) \leq N(\lambda)
$$

Hence,

$$
\frac{N(\lambda)}{\lambda^{1 / 2}} \geq \frac{N_{D}\left(\lambda,\left[a, T_{\varepsilon}\right]\right)}{\lambda^{1 / 2}} \geq \frac{1}{\pi} \int_{a}^{T_{\varepsilon}} q^{1 / 2}(t) d t-\frac{\varepsilon}{2} \geq \frac{1}{\pi} \int_{a}^{\infty} q^{1 / 2}(t) d t-\varepsilon
$$

for every $\lambda \geq \lambda(\varepsilon)$, and the proof is finished.

Remark 3.3. Let us note that Proposition 3.2 is valid whenever $\int_{a}^{\infty} q^{1 / 2}(t) d t<+\infty$, which is guaranteed by Lemma 3.1, without any monotonicity assumption. 
Proposition 3.4. Let $N(\lambda)$ be the eigenvalue counting function of Theorem 1.1. The following inequality holds:

$$
\frac{4 \lambda^{1 / 2}}{\pi} \int_{a}^{+\infty} q^{1 / 2}(t) d t+o\left(\lambda^{1 / 2}\right) \geq N(\lambda)
$$

Proof. We need a lower bound for eigenvalues due to Nehari [4]. Let $q(t)$ be a monotonic function, and $\mu_{k}$ the $k$ th Dirichlet eigenvalue of $(1.1)$ in $(a, b)$. Then,

$$
\mu_{k}\left(\int_{a}^{b} q^{1 / 2}(t) d t\right)^{2} \geq \frac{\pi^{2} k^{2}}{4} .
$$

Let $\left\{\lambda_{k}\right\}_{k \geq 0}$ be the nonprincipal eigenvalues of problem (1.1)-(1.2), and let $t_{k}$ be the $k$ th zero of the associated eigenfunction $x_{k}(t)$. Clearly, $\lambda_{k}$ coincides with the $k$ th Dirichlet eigenvalue in $\left(a, t_{k}\right)$.

Hence,

$$
\lambda_{k} \geq \frac{\pi^{2} k^{2}}{4\left(\int_{a}^{t_{k}} q^{1 / 2}(t) d t\right)^{2}} \geq \frac{\pi^{2} k^{2}}{4\left(\int_{a}^{\infty} q^{1 / 2}(t) d t\right)^{2}} .
$$

We obtain

$$
\begin{aligned}
N(\lambda) & =\#\left\{k: \lambda_{k} \leq \lambda\right\} \\
& \leq \#\left\{k: \frac{\pi^{2} k^{2}}{4\left(\int_{a}^{\infty} q^{1 / 2}(t) d t\right)^{2}} \leq \lambda\right\} \\
& =\#\left\{k: k \leq \frac{2 \lambda^{1 / 2}}{\pi} \int_{a}^{\infty} q^{1 / 2}(t) d t\right\} \\
& \leq \frac{2 \lambda^{1 / 2}}{\pi} \int_{a}^{\infty} q^{1 / 2}(t) d t+O(1),
\end{aligned}
$$

and the proof is finished.

Now we prove Theorem 1.1.

Proof of Theorem 1.1. Let be $T_{\varepsilon}$ such that

$$
\int_{T_{\varepsilon}}^{+\infty} q^{1 / 2}(t) d t<\varepsilon
$$

Applying Theorem 2.1 we obtain

$$
N(\lambda) \sim N\left(\lambda,\left(a, T_{\varepsilon}\right)\right)+N\left(\lambda,\left(T_{\varepsilon}, \infty\right)\right) .
$$

The asymptotic behavior of $N\left(\lambda,\left(a, T_{\varepsilon}\right)\right)$ is obtained from the classical theory,

$$
N\left(\lambda,\left(a, T_{\varepsilon}\right)\right) \sim \frac{\lambda^{1 / 2}}{\pi} \int_{a}^{T_{\varepsilon}} q^{1 / 2}(t) d t
$$


Hence, for $\lambda \geq \lambda(\varepsilon)$, we have

$$
N\left(\lambda,\left(a, T_{\varepsilon}\right)\right) \leq \frac{\lambda^{1 / 2}}{\pi} \int_{a}^{T_{\varepsilon}} q^{1 / 2}(t) d t+\varepsilon \lambda^{1 / 2} \leq \frac{\lambda^{1 / 2}}{\pi} \int_{a}^{+\infty} q^{1 / 2}(t) d t+\varepsilon \lambda^{1 / 2} .
$$

Now, $N\left(\lambda,\left(T_{\varepsilon}, \infty\right)\right)$ can be bounded by using Proposition 3.4:

$$
N\left(\lambda,\left(T_{\varepsilon}, \infty\right)\right) \leq \frac{2 \lambda^{1 / 2}}{\pi} \int_{T_{\varepsilon}}^{+\infty} q^{1 / 2}(t) d t \leq \varepsilon \frac{2 \lambda^{1 / 2}}{\pi} .
$$

Hence,

$$
N(\lambda) \leq \frac{\lambda^{1 / 2}}{\pi} \int_{a}^{+\infty} q^{1 / 2}(t) d t+\varepsilon \lambda^{1 / 2}+\varepsilon \frac{2 \lambda^{1 / 2}}{\pi} .
$$

Since $\varepsilon$ is arbitrarily small, and by using Proposition 3.2, we have the asymptotic expansion

$$
N(\lambda) \sim \frac{\lambda^{1 / 2}}{\pi} \int_{a}^{+\infty} q^{1 / 2}(t) d t
$$

Finally, from (3.22), we have

$$
k=N\left(\lambda_{k-1}\right) \sim \frac{\lambda_{k}^{1 / 2}}{\pi} \int_{a}^{\infty} q^{1 / 2}(t) d t
$$

which gives the asymptotic behavior of the $k$ th-eigenvalue,

$$
\lambda_{k}=\left(\frac{\pi k}{\int_{a}^{\infty} q^{1 / 2}(t) d t}\right)^{2}+o\left(k^{2}\right) .
$$

This completes the proof.

\section{Acknowledgments}

The author is supported by ANPCyT PICT No. 03-05009 and Fundacion Antorchas.

\section{References}

[1] R. Courant and D. Hilbert, Methods of Mathematical Physics. Vol. I, Interscience, New York, 1953.

[2] Á. Elbert, K. Takaŝi, and M. Naito, Singular eigenvalue problems for second order linear ordinary differential equations, Archivum Mathematicum (Brno) 34 (1998), no. 1, 59-72.

[3] P. Hartman, On the eigenvalues of differential equations, American Journal of Mathematics 73 (1951), no. 3, 657-662.

[4] Z. Nehari, Some eigenvalue estimates, Journal d'Analyse Mathématique 7 (1959), 79-88.

Juan Pablo Pinasco: Instituto de Ciencias, Universidad Nacional de General Sarmiento, J. M. Gutierrez 1150, Los Polvorines, Buenos Aires 1613, Argentina

E-mail address: jpinasco@ungs.edu.ar 


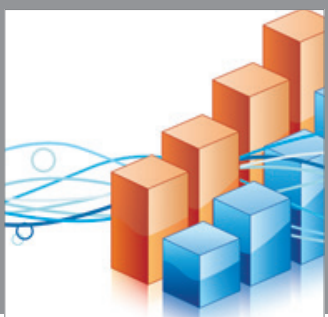

Advances in

Operations Research

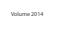

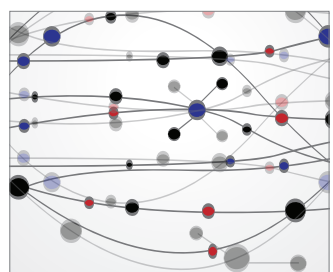

\section{The Scientific} World Journal
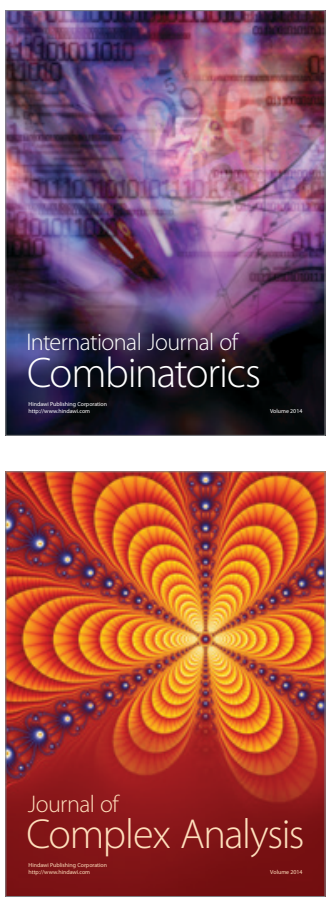

International Journal of

Mathematics and

Mathematical

Sciences
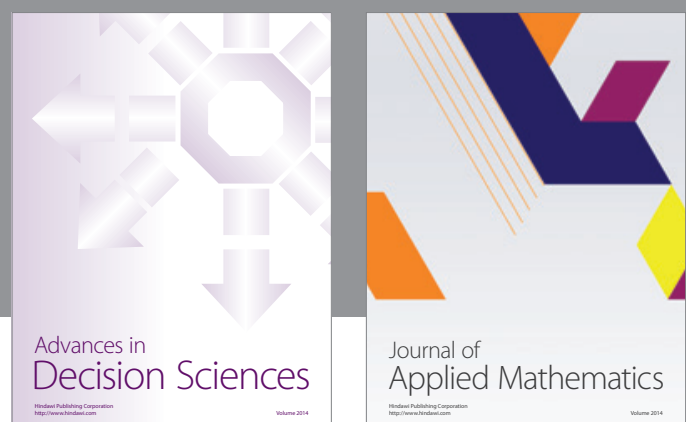

Journal of

Applied Mathematics
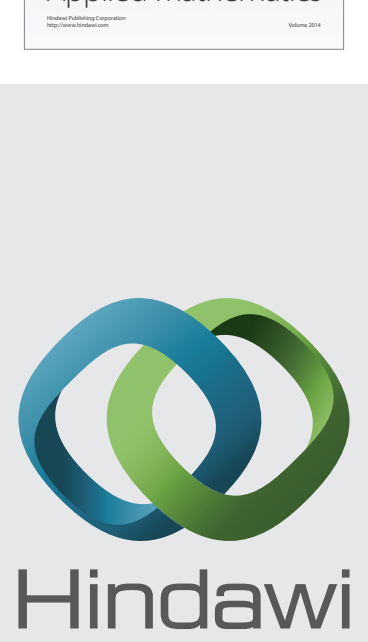

Submit your manuscripts at http://www.hindawi.com
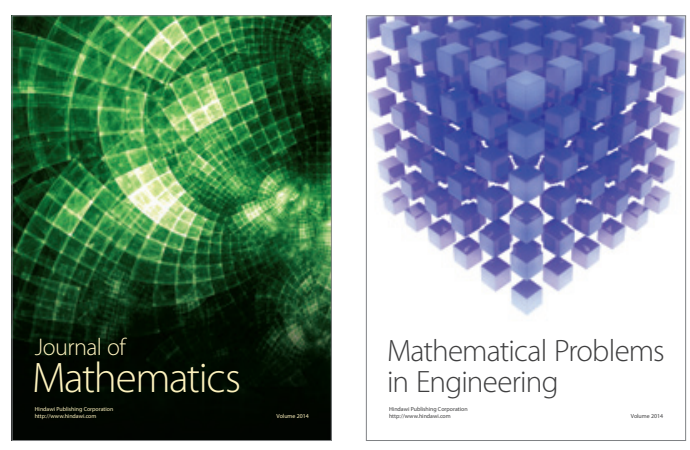

Mathematical Problems in Engineering
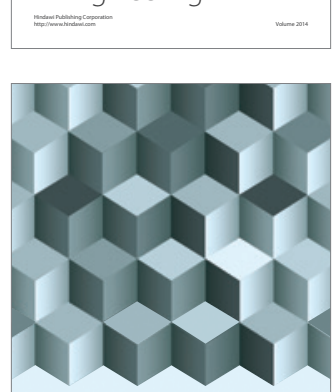

Journal of

Function Spaces
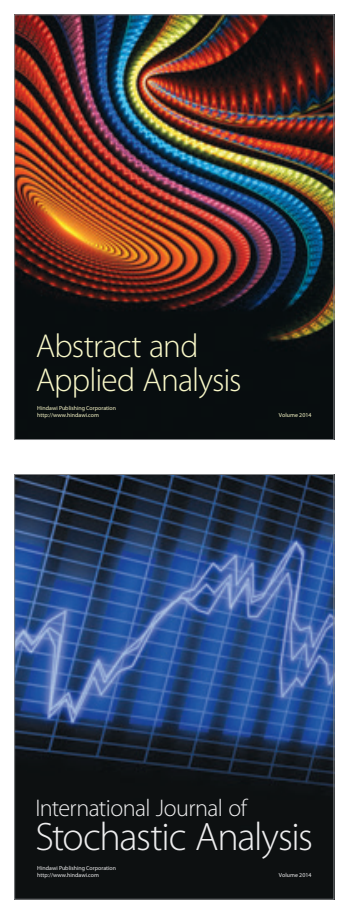

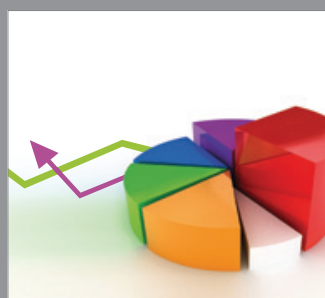

ournal of

Probability and Statistics

Promensencen
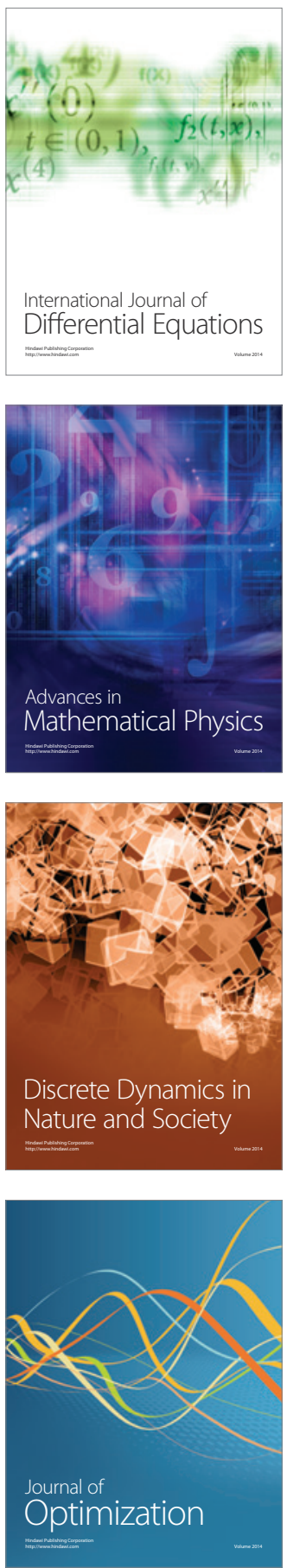